\title{
Analysis of Draft Genome Resources of Thirty-Three Canadian Strains of Pseudomonas syringae pv. tomato Isolated Between 1992 and 2008 Reveals Achromobactin Virulence Cluster that Is Absent in the Reference Strain DC3000
}

\author{
James T. Tambong, ${ }^{1,2, \dagger}$ Renlin Xu, ${ }^{1}$ Diane Cuppels, ${ }^{3}$ Julie Chapados, ${ }^{1}$ Suzanne Gerdis, ${ }^{1}$ \\ Jackson Eyres, ${ }^{4}$ Adam Koziol, ${ }^{5}$ and Jeremy Dettman ${ }^{1}$ \\ ${ }^{1}$ Ottawa Research and Development Centre, Agriculture and Agri-Food Canada, Ottawa, Ontario, Canada \\ ${ }^{2}$ Department of Plant Science, University of Manitoba, Winnipeg, Manitoba, Canada \\ ${ }^{3}$ London Research and Development Centre, Agriculture and Agri-Food Canada, London, Ontario, Canada \\ (Retired) \\ ${ }^{4} \mathrm{BICoE}$, Agriculture and Agri-Food Canada, Ottawa, Ontario, Canada \\ ${ }^{5}$ Canadian Food Inspection Agency, Ottawa, Ontario, Canada
}

\section{Genome Resource Announcement}

Pseudomonas syringae pv. tomato (Okabe 1933) Young et al. 1978 is the causal agent of bacterial speck disease of field and greenhouse tomato plants, and is an economically important disease of tomato (Lycopersicon esculentum). Only one Canadian whole-genome sequence of this economically important pathogen has been publicly available in NCBI GenBank. In 1992 to 2008, annual surveys of tomato fields and greenhouses were conducted in Ontario, Canada, and leaves, stems, and fruit exhibiting typical symptoms of the bacterial disease were collected. The pathogen was isolated from diseased plant material using Vogel-Bonner-tartrate agar, as previously described (Cuppels et al. 1990). Purified strains of the pathogen were stored at $-80^{\circ} \mathrm{C}$ in nutrient broth-yeast extract broth plus $15 \%$ glycerol (Cuppels et al. 1990). The bacterial isolates were cultured overnight in Luria-Bertani broth and the genomic DNAs were extracted using the DNeasy UltraClean Microbial kit (Qiagen), with minor modifications. Briefly, $600 \mu \mathrm{l}$ of microbial culture was used to generate a cell pellet, which was stored at $-20^{\circ} \mathrm{C}$ until used, and $36 \mu \mathrm{l}$ of RNase Cocktail (Invitrogen) was added to the cell suspension before processing. Also, homogenization was performed using a Tissue Lyser II (Qiagen) set to $20 \mathrm{~Hz}$ for $5 \mathrm{~min}$, after which the process was repeated once with plates reoriented at $180^{\circ}$ prior to incubation at $37^{\circ} \mathrm{C}$ for $30 \mathrm{~min}$. The isolates were characterized to the genus Pseudomonas using 16S ribosomal RNA (rRNA) sequencing (Lane 1991; Tambong et al. 2017) and BLAST analysis (Altschul et al. 1990). We report, here, draft whole genome sequences of 33 Pseudomonas syringae pv. tomato (Pst) strains. Libraries were constructed using a Nextera DNA Flex prep kit (Illumina) following the manufacturer's instructions. The draft genome sequences were determined by paired-end sequencing using Illumina NextSeq technology at the Molecular Technologies Laboratory (Ottawa Research and Development Centre, Ottawa, Canada). The mean numbers of pairedend reads of 2,020,245 bp (1,314,234 to 2,955,580 bp), each $150 \mathrm{bp}$ long, were generated (Table 1). The quality of the reads was checked using FastQC (Andrews 2010), trimmed using BBDUK (Bushnell 2014), if required, and de novo assemblies were performed using SKESA (Souvorov et al. 2018), as implemented in COWBAT ver. 0.5.0.7 pipeline (https://github.com/OLCBioinformatics/COWBAT). Scaffolds $<500$ bp long were discarded. The best assemblies, based

\footnotetext{
${ }^{\dagger}$ Corresponding author: J. T. Tambong; james.tambong@agr.gc.ca
}

The author(s) declare no conflict of interest.

Accepted for publication 17 September 2021
Funding

Support for genome sequencing and assembly of these bacterial strains was provided by Agriculture and Agri-Food Canada through the Biological Collections Data Mobilization Initiative (BioMob, Work Package 2, J-001564); and comparative genomics analysis was funded through projects $\mathrm{J}-002272$, $\mathrm{J}-001577$, and J-000409.

Keywords bacterial pathogens, evolution, genomics, microbe-genome sequencing 
Table 1. Basic genome statistics of the 33 Canadian strains of Pseudomonas syringae pv. tomato isolated from 1992 to $2008^{a}$

\begin{tabular}{|c|c|c|c|c|c|c|c|c|c|c|c|c|}
\hline Strains & $\begin{array}{l}\text { Year of } \\
\text { isolation }\end{array}$ & $\begin{array}{l}\text { Total } \\
\text { reads }\end{array}$ & Depth $^{\text {b }}$ & Contigs & $N_{50}(b p)$ & $\mathrm{L}_{50}$ & $\begin{array}{c}\text { Largest } \\
\text { contig (bp) }\end{array}$ & $\begin{array}{c}\text { Total } \\
\text { length (bp) }\end{array}$ & GC (\%) & $\mathrm{CDSs}^{\mathrm{c}}$ & \multicolumn{2}{|c|}{ Protein-encoding genes } \\
\hline DC 92-1 & 1992 & $2,282,872$ & 53 & 132 & 133,701 & 17 & 369,737 & $6,323,733$ & 58.59 & 6,075 & 3,933 & 2,142 \\
\hline DC $92-7$ & 1992 & $1,852,375$ & 42 & 143 & 176,505 & 12 & 574,824 & $6,358,250$ & 58.59 & 6,104 & 3,957 & 2,147 \\
\hline DC 93-1 & 1993 & $1,957,327$ & 45 & 116 & 141,037 & 15 & 356,731 & $6,300,490$ & 58.62 & 6,024 & 3,910 & 2,114 \\
\hline DC 94-1 & 1994 & $1,871,646$ & 43 & 119 & 138,516 & 15 & 298,064 & $6,271,099$ & 58.62 & 5,993 & 3,909 & 2,084 \\
\hline DC 95-2 & 1995 & $2,850,576$ & 67 & 100 & 153,003 & 13 & 371,446 & $6,214,671$ & 58.60 & 5,913 & 3,868 & 2,045 \\
\hline DC 96-2 & 1996 & $2,396,612$ & 55 & 170 & 159,324 & 14 & 574,677 & $6,381,632$ & 58.55 & 6,166 & 3,968 & 2,198 \\
\hline DC $96-3$ & 1996 & $2,557,587$ & 60 & 91 & 164,584 & 14 & 291,886 & $6,199,279$ & 58.62 & 5,901 & 3,878 & 2,023 \\
\hline DC 97T23A & 1997 & $2,274,160$ & 53 & 136 & 138,127 & 14 & 390,693 & $6,304,758$ & 58.62 & 6,048 & 3,920 & 2,128 \\
\hline DC 97T24A & 1997 & $2,540,768$ & 59 & 149 & 150,184 & 15 & 335,638 & $6,303,202$ & 58.61 & 6,050 & 3,925 & 2,125 \\
\hline DC 98-1 & 1998 & $1,950,553$ & 45 & 108 & 168,384 & 14 & 401,594 & $6,363,517$ & 58.56 & 6,072 & 3,926 & 2,146 \\
\hline DC 00T2C & 2000 & $1,955,228$ & 45 & 121 & 138,905 & 14 & 453,565 & $6,322,847$ & 58.59 & 6,044 & 3,925 & 2,119 \\
\hline DC 00ТЗA & 2000 & $2,213,642$ & 51 & 133 & 150,137 & 13 & 356,719 & $6,317,806$ & 58.61 & 6,053 & 3,921 & 2,132 \\
\hline DC 00T4A & 2000 & $2,184,112$ & 50 & 168 & 127,738 & 15 & 369,718 & $6,390,924$ & 58.55 & 6,160 & 3,979 & 2,181 \\
\hline DC 01T23B2 & 2001 & $1,471,655$ & 34 & 156 & 149,304 & 14 & 389,356 & $6,341,373$ & 58.56 & 6,097 & 3,949 & 2,148 \\
\hline DC 01T30A & 2001 & $1,639,263$ & 38 & 156 & 126,851 & 16 & 322,567 & $6,342,470$ & 58.56 & 6,044 & 3,925 & 2,119 \\
\hline DC 01T31C & 2001 & $1,314,234$ & 30 & 162 & 101,231 & 20 & 291,627 & $6,308,123$ & 58.59 & 6,080 & 3,938 & 2,142 \\
\hline DC 02T10A & 2002 & $1,725,363$ & 40 & 156 & 118,097 & 15 & 575,284 & $6,326,087$ & 58.59 & 6,030 & 3,907 & 2,123 \\
\hline DC 02T2A & 2002 & $1,548,325$ & 36 & 144 & 124,077 & 17 & 291,742 & $6,282,724$ & 58.60 & 6,085 & 3,950 & 2,135 \\
\hline DC 03 T12 & 2003 & $1,808,136$ & 42 & 129 & 126,813 & 15 & 385,340 & $6,284,879$ & 58.59 & 6,008 & 3,900 & 2,108 \\
\hline DC 03T6 & 2003 & $1,661,778$ & 38 & 132 & 149,283 & 13 & 356,698 & $6,283,754$ & 58.60 & 6,017 & 3,915 & 2,102 \\
\hline DC 04T2A & 2004 & $1,851,271$ & 43 & 145 & 126,813 & 15 & 385,256 & $6,323,422$ & 58.59 & 6,050 & 3,929 & 2,121 \\
\hline DC 04T2B & 2004 & $1,910,866$ & 44 & 135 & 139,077 & 14 & 402,377 & $6,303,383$ & 58.60 & 6,014 & 3,922 & 2,092 \\
\hline
\end{tabular}

${ }^{a}$ Whole-genome sequences were generated using Illumina NextSeq platform and assembled using SKESA. Contigs <500 bp were discarded. Based on CheckM algorithm (Parks et al. 2015) as implemented in PATRIC (Wattam et al. 2014), the completeness of the genomes was $100 \%$, with coarse consistency of 99.3 to $99.7 \%$ and fine consistency of 97.3 to $97.7 \%$. Annotation and protein function assignment were implemented in PATRIC (Wattam et al. 2014).

${ }^{\mathrm{b}}$ Average coverage depth.

${ }^{c}$ Total number of protein-coding sequences.

${ }^{d}$ Protein-encoding genes with and without functional assignment.

on $\mathrm{N}_{50}$ and $\mathrm{L}_{50}$ values and nearness to the expected genome size, were obtained. The average size of the draft genome sequences is $6.305 \mathrm{Mbp}$ with a range of 6.195 to $6.390 \mathrm{Mbp}$ (average maximum contig $=381,352 \mathrm{bp}$ and $N_{50}=139,729 \mathrm{bp}$ ) (Table 1). The average $\mathrm{G}+\mathrm{C}$ content of the draft genome sequences is $58.59 \%$ (58.55 to $58.65 \%)$, with an average estimated coverage of $46.8 \times(36 \times$ to $67 \times)$.

The 33 draft genome sequences had a completeness of $100 \%$, average consistency of $97.6 \%$, coarse consistency of $99.6 \%$, and average contamination rate of $<0.5 \%$ based on the CheckM algorithm (Parks et al. 2015) as implemented in PATRIC pipeline version 3.6.9 (Pathosystems Resource Integration Center) (Wattam et al. 2014). The whole-genome sequences exhibited average nucleotide identity (Jain et al. 2018) values of 98.64 to $98.72 \%$ with P. syringae pv. tomato ICMP $2844^{\mathrm{PT}}$ or DC3000, validating the taxonomic standing of these Canadian strains. The assembled sequences were annotated using the RAST tool kit (RASTtk) (Brettin et al. 2015) as implemented in the PATRIC pipeline, which identified 5,889 to 6,166 protein-coding sequences (CDSs). The 33 genome sequences, each had 50 to 58 transfer RNAs, 2 to 6 rRNAs, and 1 transfer-messenger RNA based on Prokka version 1.14.5 (Seemann 2014). Default parameters were used for all software unless otherwise specified. The Roary pipeline (Page et al. 2015) was used to compute pan and core genomes of 6,808 (Fig. 1A) and 4,993 (Fig. 1B) CDSs, respectively. A pan-genome single-nucleotide polymorphism-based tree clustered the strains into distinct groups (Fig. 1A). Strain Pst DC 98-1 

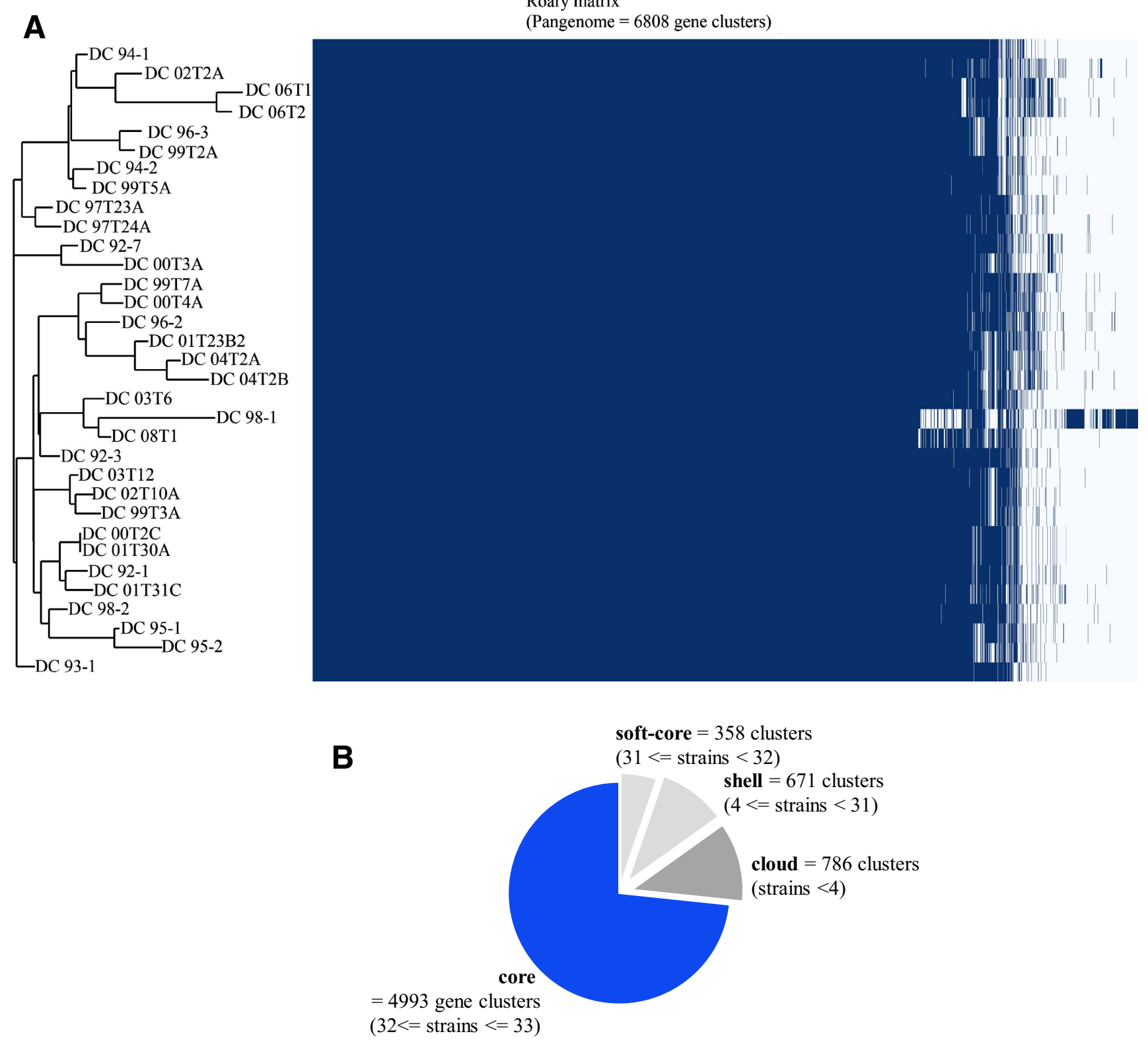

Fig. 1. A, Pan-genome single-nucleotide polymorphism (panSNP) tree and Roary pangenome matrix and B, pie chart of core-genomes of the 33 Canadian strains of Pseudomonas syringae pv. tomato based on BLAST as implemented in Roary pipeline (Page et al. 2015). Taxa names on the panSNP tree are the strain codes without the prefix "Pst".

showed a unique distribution pattern of the gene clusters, suggesting the presence of several accessory genes (Fig. 1A). Cloud (accessory), shell, and soft-core gene clusters of $786(<4$ strains; for example, Pst DC 98-1), $671(4 \leq$ strains $<31)$, and 358 ( $31 \leq$ strains $<32)$, respectively, were identified (Fig. 1B). Genome mining for virulence factors using the virulence factors of pathogenic bacteria tool (Liu et al. 2019) identified adherence-related genes (e.g., flagella, type IV pili biosynthesis, and lipopolysaccharide O-antigen), CDSs implicated in antiphagocytosis (e.g., alginate biosynthesis or regulation), secretion system apparatuses, and effectors. Also, complete or partial pyoverdine and achromobactin siderophore biosynthetic clusters and iron transport genes were identified in all the strains (Table 2). The achromobactin biosynthetic cluster was not detected in P. syringae pv. tomato DC3000 (Table 2) or ICMP 2844 (pathotype). This suggests that the Canadian $P$. syringae pv. tomato strains might have acquired the achromobactin biosynthetic cluster and iron transport genes from another bacterial group, possibly $P$. syringae pv. syringae. An additional iron-chelating siderophore in the Canadian strains could significantly improve their ecological fitness in iron-limiting niches. A complete (20 genes) or partial cluster of genes for the coronatine phytotoxin, reported to be involved in pathogenicity on tomato, was identified in all strains except Pst DC 95-1, Pst DC 
Table 2. Achromobactin biosynthetic, coronatine phytotoxin (Cor), lipopolysaccharide O-antigen (LPS), and type I O-polysaccharide (Cap1) genes detected in the 33 whole genome sequences of the Canadian strains of Pseudomonas syringae pv. tomato ${ }^{a}$

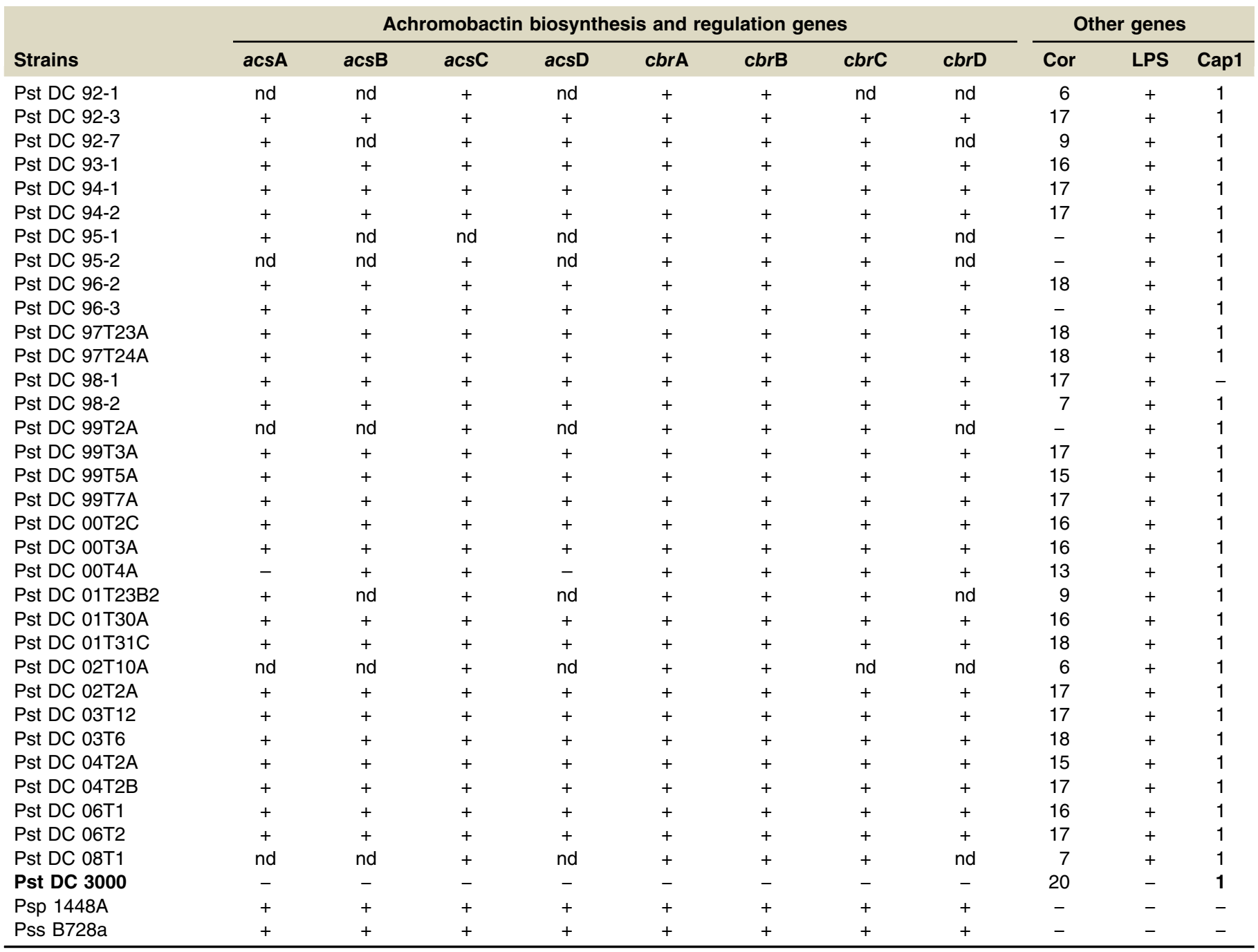

${ }^{a}$ Pst $=P$. syringae pv. tomato, $\mathrm{Psp}=P$. syringae pv. phaseolicola, Pss $=P$. syringae pv. syringae, + indicates gene is present, - indicates gene not present, and $n d=$ not detected. For coronatine phytotoxin, numbers indicate number of genes present out of the expected 20 genes. Note the absence of achromobactin biosynthetic cluster in the complete genome sequence of strain DC3000. Virulence factors were detected using the virulence factors of pathogenic bacteria tool (Liu et al. 2019).

95-2, Pst DC 96-3, and Pst DC 99T2A (Table 2). These whole-genome data of Canadian strains of $P$. syringae pv. tomato could be useful resources in understanding the pathogenicity and evolution of this pathogen over time.

\section{Data Availability}

The whole-genome sequences are deposited in DNA Data Bank of Japan/European Nucleotide Archive/GenBank under the accession numbers JAIFYB000000000 to JAIFZH000000000. The raw reads are deposited in the NCBI Sequence Read Archive under the accession number PRJNA754202.

\section{Acknowledgments}

We thank the staff of Molecular Technologies Laboratory, Ottawa Research and Development Centre, Ottawa, Canada (MTL-ORDC), for the Illumina NextSeq sequencing; the London Research and Development Centre for resources provided to D. Cuppels (retired) to conduct these surveys; and all of the summer students and technicians at ORDC and London Research and Development Centre who worked on these projects. 


\section{Literature Cited}

Altschul, S. F., Gish, W., Miller, W., Myers, E. W., and Lipman, D. J. 1990. Basic local alignment search tool. J. Mol. Biol. 215:403-410.

Andrews, S. 2010. FastQC: A quality control tool for high throughput sequence data. https://www.bioinformatics.babraham.ac.uk/projects/fastqc/

Brettin, T., Davis, J., Disz, T., Edwards, R. A., Gerdes, S., Gary, J., Olsen, G., Olson, R., Overbeek, R., Parrello, B., Pusch, G. D., Shukla, M., Thomason, J. A., III, Stevens, R., Vonstein, V., Wattam, A. R., and Xia, F. 2015. RASTtk: A modular and extensible implementation of the RAST algorithm for building custom annotation pipelines and annotating batches of genomes. Sci. Rep. 5: 8365.

Bushnell, B. 2014. BBTools software package. https://jgi.doe.gov/data-and-tools/ bbtools/

Cuppels, D. A., Moore, R. A., and Morris, V. L. 1990. Construction and use of a nonradioactive hybridization probe for detection of Pseudomonas syringae pv. tomato on tomato plants. Appl. Environ. Microbiol. 56:1743-1749.

Jain, C., Rodriguez, R. L., Phillippy, A. M., Konstantinidis, K. T., and Aluru, S. 2018. High throughput ANI analysis of $90 \mathrm{~K}$ prokaryotic genomes reveals clear species boundaries. Nat. Commun. 9:5114.

Lane, L. D. 1991. 16S/23S rRNA sequencing. Pages 115-175 in: Nucleic Acid Techniques in Bacterial Systematics. E. Stackebrandt and M. Goodfellow, eds. John Wiley \& Sons, Ltd., Chichester, England.
Liu, B., Zheng, D. D., Jin, Q., Chen, L. H., and Yang, J. 2019. VFDB 2019: A comparative pathogenomic platform with an interactive web interface. Nucleic Acids Res. 47:D687-D692.

Page, A. J., Cummins, C. A., Hunt, M., Wong, V. K., Reuter, S., Holden, M. T. G., Fookes, M., Falush, D., Keane, J. G., and Parkhill, J. 2015. Roary: Rapid largescale prokaryote pan genome analysis. Bioinformatics 31:3691-3693.

Parks, D. H., Imelfort, M., Skennerton, C. T., Hugenholtz, P., and Tyson, G. W. 2015. Assessing the quality of microbial genomes recovered from isolates, single cells, and metagenomes. Genome Res. 25:1043-1055.

Seemann, T. 2014. Prokka: Rapid prokaryotic genome annotation. Bioinformatics 30 : 2068-2069.

Souvorov, A., Agarwala, R., and Lipman, D. J. 2018. SKESA: Strategic k-mer extension for scrupulous assemblies. Genome Biol. 19:153.

Tambong, J. T., Xu, R., and Bromfield, E. 2017. Pseudomonas canadensis sp. nov., a biological control agent isolated from a field plot under long-term mineral fertilization. Int. J. Syst. Evol. Microbiol. 67:889-895.

Wattam, A. R., Abraham, D., Dalay, O., Disz, T. L., Driscoll, T., Gabbard, J. L., Gillespie, J. J., Gough, R., Hix, D., Kenyon, R., Machi, D., Mao, C., Nordberg, E. K., Olson, R., Overbeek, R., Pusch, G. D., Shukla, M., Schulman, J., Stevens, R. L., Sullivan, D. E., Vonstein, V., Warren, A., Will, R., Wilson, M. J., Yoo, H. S., Zhang, C., Zhang, Y., and Sobral, B. W. 2014. PATRIC, the bacterial bioinformatics database and analysis resource. Nucleic Acids Res. 42:D581-D591. 